\title{
Present Situation of Electrical Energy Demand, Supply, Projection of Demand-supply and Renewable Energy Utilization and Progress
}

\author{
Md. Nazmul Islam \\ Department of Electrical and Electronic Engineering \\ Bangabandhu Sheikh Mujibur Rahman Science \& Technology University Gopalganj, Bangladesh \\ nazmulislam1581@gmail.com
}

\begin{abstract}
A brief overview of present electricity demand, supply, projection of them and renewable energy generation and progress has been presented in this paper. Electricity is the major source of power for most of the country's economic activities. According to the Bangladesh Power Development Board in July 2018, 90 percent of the population had access to electricity. However per capita energy consumption in Bangladesh is considered low. The country has a very limited energy reserve; small amounts of oil, coal and countable natural gas reserves. The country suffers an internal energy struggle, as about $88 \%$ of the country's power producing thermal plants are gas-based. To solve this problem, renewable energy is being used.
\end{abstract}

Keywords-Electrical Energy Supply, Energy Demand, Present Situation, Projection Supply and Demand, Consumption Pattern, Renewable Energy, Distribution loss.

\section{INTRODUCTION}

Electricity is the major source of power for most of the country's economic activities.Bangladesh being a developing country, the electric energy demand has been increasing at a significantly high rate as well as electric energy supply has been increasing but the state-owned electricity utilities suffer from large energy shortages. The electricity Demand in the system varies one hours to other hours. The maximum demand is occurred during $5 \mathrm{pm}$ to $11 \mathrm{pm}$ which is termed as 'peak hour' and other part of the time is termed as off-peak hour. The extent of this variation is measured in terms of Load Factor, which is the ratio of average and maximum demand. For economic reasons, it is desirable to have a higher Load Factor, as this would permit better utilization of plant capacity. Moreover, the cost of energy supply during peak hour is higher, because some relatively costlier power plants are required to put in operation during the peak hour. For these reasons, load management is essential throughout the year for better capacity utilization of power plants and minimum generation cost.

In the time of Nawab Khwaja Ahsanullah, electrification of the capital city Dhaka began in 1901, only 19 years after New York and 13 years after London. [2] Later, an Electricity Directorate was established in East Pakistan in 1948. There were few Steam Turbines in Siddhirganj (maximum size 10MW), Chittagong and Khulna area at that time. In 1962, 40 MW Kaptai hydroelectric project was installed a $132 \mathrm{kV}$ transmission line between Dhaka and Chittagong was commissioned. [8] In 1971, after the independence of Bangladesh only $3 \%$ of the population had access to electricity. In 2010, Shikalbaha and Siddhirganj power plant start to generated $255 \mathrm{MW}$ and also start to generated $520 \mathrm{MW}$ in some private sector. About 1763MW electricity add in national grid and captive power. It has been increased to $59.6 \%$ in 2012. However, $79 \%$ percent of the connected consumers suffer from load-shedding, and $60 \%$ of the consumers face low voltage supply. In 1974-75, the installed electricity generation capacity was $667 \mathrm{MW}$ whereas in August 2016 it was $12780 \mathrm{MW}$ including the 600 MW power import from India. Bangladesh's total installed electricity generation capacity (including captive power) was 15,351 megawatts (MW) as of January

2017[4] and 15,500-megawatts in 2018[6]. The $88 \%$ of the population had access to electricity in 2018. [6] There are 52 public power plant in 2019 which generated about 8,845-Megawatt electricity. 
The total installed capacity is 20,000 MW (combining solar power). Bangladesh's energy sector is booming.

The present government is committed to ensuring access to affordable and reliable electricity for all citizens by 2021. Recently, Bangladesh started construction of the 2.4gigawatt (GW) Rooppur Nuclear Power Plant expected to go into operation in 2023. Also start construction of 7,554 MW power plant (such as Matarbari Power Station, Payra Thermal Power Plant, Rampal Power Plant, Feni Lanka Power Limited, Payra LNG Power Plant). Bangladesh will need an estimated 35,000 MW power by 2030. Bangladesh Government has undertaken various projects to meet this rapidly growing demand. They propose some new power plant. Our government is expecting to fill-up all shortage of electricity supply after completing these projects.

\section{ELECTRICAL ENERGY SYSTEM IN BANGLADESH}

Electrical energy system discusses about mainly generation \& supply sector, demand sector, consumption sector. The supply sector is categorical as commercial energy and biomass fuels. The commercial energy comprises of natural gas, oil and electricity while the biomass fuels are agricultural wastes, animal wastes and fuel wood. In the demand sector, energy consumers make decision to utilize gas, oil and electricity based on both fuel price and the availability of the fuels whereas the biomass fuel consumption is mainly based on the availability of biomass fuels. The supply sectors (commercial and biomass) are the fuel supplies and imports. The imports are equal to the shortfalls in domestic supply.

\section{A. Electrical Energy Generation}

The power sector has experienced considerable progress in meeting the demand for electricity. Access, coverage, and level of consumption have significantly increased over the years. Daily load shedding has significantly dropped. Natural gas and coal are the main sources for power generation in the country. About $62.9 \%$ of Bangladeshi generated electricity comes from natural gas, while $10 \%$ is from diesel, $5 \%$ comes from coal, $3 \%$ of heavy oil, and $3.3 \%$ is of renewable sources.[2] There also some power plants are under construction and some are proposed. Total installed capacity was $15,953 \mathrm{MW}$ which includes 4,452 MW IPP/SIPP, 1745 MW Rental Power Plant \& $251 \mathrm{MW}$ in REB (for PBS) and 660MW Power Import from India. The maximum peak generation was $10,958 \mathrm{MW}$ which was $15.60 \%$ higher than that in the previous year. The reasons for lower peak generation with respect to generation capacity were: (i) some plants are out of operation for maintenance, rehabilitation \& overhauling (ii) capacity of some plants dated due to aging and (iii) gas shortage.

Total net energy generation in FY 2018 was $62,678 \mathrm{GWh}$, which was about $9.43 \%$ higher than previous year's net generation of 57,276 GWh. Net energy generation in the public sector was $31,082 \mathrm{GWh}$ and $26,813 \mathrm{GWh}$ in the private sector (including REB). Another 4,783 $\mathrm{GWh}$ was imported from India through the interconnection in Bheramara and Tripura.

The list of power plants in Bangladesh is given below: 
Table-1: List of all power plant in Bangladesh [3,11]

\begin{tabular}{|c|c|c|c|}
\hline SL No & Name of power plant & $\begin{array}{c}\text { Type } \\
\text { of fuel }\end{array}$ & $\begin{array}{c}\text { Installed Capacity (As of June) } \\
\text { (MW) }\end{array}$ \\
\hline \multirow[t]{4}{*}{1} & Ghorashal 2x55 MW ST & Gas & 110 \\
\hline & Ghorashal 2x210 MW ST & Gas & 420 \\
\hline & Ghorashal 210 MW ST (5th Unit) & Gas & 210 \\
\hline & Ghorashal $210 \mathrm{MW} 7^{\text {th }}$ & Gas & 365 \\
\hline 2 & Tongi 105MW GT & Gas & 105 \\
\hline 3 & Haripur 3x33 MW GT & Gas & 64 \\
\hline 4 & Siddhirganj 210 MW ST & Gas & 210 \\
\hline 5 & Siddhirganj 2x120 MW GT & Gas & 210 \\
\hline 6 & Haripur $412 \mathrm{MW}$ CCPP & Gas & 412 \\
\hline 7 & RPCL Gazipur $52 \mathrm{MW}$ & F.oil & 52 \\
\hline 8 & Kodda Gazipur $150 \mathrm{MW}$ (PDB-RPCL) & F.oil & 149 \\
\hline 9 & Siddhirganj 335 MW CCPP (EGCB) & Gas & 217 \\
\hline 10 & Karnafuli Hydro & Hydro & 230 \\
\hline \multirow[t]{2}{*}{11} & Rauzan 210 MW /ST (1st) & Gas & 210 \\
\hline & Rauzan 210 MW /ST (2nd) & Gas & 210 \\
\hline 12 & Chattogram $60 \mathrm{MW} / \mathrm{ST}$ & Gas & 60 \\
\hline \multirow[t]{2}{*}{13} & \multirow[t]{2}{*}{ Shikalbaha 150 MW Peaking PP } & Gas & \multirow[t]{2}{*}{150} \\
\hline & & HSD & \\
\hline 14 & Hathazari $100 \mathrm{MW}$ Peaking PP & F.oil & 98 \\
\hline 15 & Sangu, Dohazari-kaliaish 100 MW PPP & F.oil & 102 \\
\hline 16 & RPCL Raozan $25 \mathrm{MW}$ & F.oil & 25 \\
\hline \multirow[t]{2}{*}{17} & \multirow[t]{2}{*}{ Shikalbaha 225 MW PS } & Gas & \multirow[t]{2}{*}{225} \\
\hline & & HSD & \\
\hline 18 & Sonagazi $1 \mathrm{MW}$ Wind PP & Gas & 0 \\
\hline 19 & Kutubdia 900KW Wind PP & Gas & 0 \\
\hline 20 & Ashuganj 2x64 MW Steam Turbine & Gas & \\
\hline 21 & Ashuganj 3x150 MW Steam Turbine & Gas & 450 \\
\hline 22 & Ashuganj $50 \mathrm{MW}$ GE & Gas & 53 \\
\hline 23 & Ashuganj $225 \mathrm{MW}$ CCPP & Gas & 221 \\
\hline 24 & Ashuganj (South) $450 \mathrm{MW}$ CCPP & Gas & 360 \\
\hline 25 & Ashuganj (North) $450 \mathrm{MW}$ CCPP & Gas & 360 \\
\hline 26 & Chandpur $150 \mathrm{MW}$ CCPP & Gas & 163 \\
\hline 27 & Titas (Doudkandi) $50 \mathrm{MW}$ RE & F.oil & 52 \\
\hline 28 & Shahjibazar 70 MW GT, Habiganj & Gas & 70 \\
\hline 29 & Shahjibazar 330 MW CCPP & Gas & 330 \\
\hline \multirow[t]{2}{*}{30} & Fenchuganj C.C. (Unit \#1) & Gas & 97 \\
\hline & Fenchuganj C.C. (Unit \#2) & Gas & 104 \\
\hline 31 & Sylhet 1x20 MW /GT & Gas & 20 \\
\hline 32 & Sylhet 1x150 MW /GT & Gas & 142 \\
\hline 33 & Khulna 1x110 MW Steam Turbine & F.oil & 0 \\
\hline 34 & Khulna 225 MW (NWPGCL) & HSD & 230 \\
\hline 35 & Bheramara 3x20 MW /GT & HSD & 60 \\
\hline 36 & Bheramara 360 MW CCPP (NWPGCL) & Gas & 410 \\
\hline 37 & Faridpur $50 \mathrm{MW}$ Peaking PP & F.oil & 54 \\
\hline 38 & Gopalganj $100 \mathrm{MW}$ Peaking PP & F.oil & 109 \\
\hline 39 & Barishal 2x20 MW /GT & HSD & 40 \\
\hline 40 & Bhola 225 MW CCPP & Gas & 194 \\
\hline \multirow[t]{2}{*}{41} & Baghabari $71 \mathrm{MW} / \mathrm{GT}$ & Gas & 71 \\
\hline & Baghabari 100 MW /GT & Gas & 100 \\
\hline \multirow[t]{2}{*}{42} & \multirow[t]{2}{*}{ Sirajgonj 210 MW CC (NWPGCL) Unit-1 } & Gas & \multirow[t]{2}{*}{210} \\
\hline & & HSD & \\
\hline 43 & Baghabari 50 MW Peaking RE & F.oil & 52 \\
\hline 44 & Bera 70 MW Peaking RE & F.oil & 71 \\
\hline 45 & Santahar $50 \mathrm{MW}$ PP & F.oil & 50 \\
\hline
\end{tabular}




\begin{tabular}{|c|c|c|c|}
\hline 46 & Katakhali 50 MW PP & Gas & 50 \\
\hline 47 & Chapainobabgonj Peaking Power Station & HSD & 104 \\
\hline \multirow[t]{2}{*}{48} & \multirow[t]{2}{*}{ Sirajgonj 210 MW CC (NWPGCL) Unit-2 } & Gas & \multirow[t]{2}{*}{220} \\
\hline & & HSD & \\
\hline \multirow[t]{2}{*}{49} & \multirow[t]{2}{*}{ Sirajgonj 210 MW CC (NWPGCL) Unit-3 } & Gas & \multirow[t]{2}{*}{0} \\
\hline & & HSD & \\
\hline \multirow[t]{2}{*}{50} & Barapukuria Coal based S/T (unit 1,2) & Coal & 250 \\
\hline & Barapukuria Coal based S/T (unit 3) & Coal & 274 \\
\hline 51 & Saidpur 20 MW /GT & HSD & 20 \\
\hline 52 & Rangpur 20 MW /GT & HSD & 20 \\
\hline & Sub-Total grid (Public) & & 8845 \\
\hline \multicolumn{4}{|c|}{ Private Sector } \\
\hline SL No. & Name & Fuel Type & Capacity (MW) \\
\hline 1. & Midland Power Co. Ashuganj 51 MW & Gas & 51 \\
\hline 2. & Rural Power Company Ltd.(RPCL) $210 \mathrm{MW}$ & Gas & 210 \\
\hline 3. & Haripur Power Ltd. (CDC) & Gas & 360 \\
\hline 4. & Meghnaghat Power Ltd. & Gas & 450 \\
\hline 5. & $\begin{array}{l}\text { Ghorashal, Regent Energy \& Power Ltd. } 108 \\
\text { MW }\end{array}$ & Gas & 108 \\
\hline 6. & $\begin{array}{c}\text { Ashuganj Modular (United Power Co. Ltd.) } 195 \\
\text { MW }\end{array}$ & Gas & 195 \\
\hline 7. & Summit Bibiyana-2 Power Co. Ltd. 341 MW & Gas & 341 \\
\hline \multirow[t]{2}{*}{8.} & Kushiara Power Co. Ltd(163 MW) CCPP & Gas & 163 \\
\hline & Fenchuganj & & \\
\hline 9. & Daudkandi 200MW(Bangla Trac.) & HSD & 200 \\
\hline 10. & Noapara 100 MW9Bangla Trac) & HSD & 100 \\
\hline 11. & $\begin{array}{c}\text { Kodda Gazipur 300MW Power Ltd.(Unit-2) } \\
\text { Summit }\end{array}$ & F. Oil & 300 \\
\hline \multirow[t]{2}{*}{12.} & $\begin{array}{c}\mathrm{KPCl} \quad(110 \mathrm{MW}), \mathrm{U}-1 \text { Tiger-1,3 (Burg) } \\
\text { BMPP }\end{array}$ & F. Oil & 110 \\
\hline & (summit-United) & & \\
\hline \multirow[t]{2}{*}{13.} & $\begin{array}{c}\text { NEPC Construction Power Ltd. (Haripur } \\
\text { BMPP) }\end{array}$ & F. Oil & 100 \\
\hline & $110 \mathrm{MW}$ & & \\
\hline 14. & Raj. Lanka Power Gen. Com Ltd. 55 MW, Natore & F. Oil & 52 \\
\hline 15. & Summit Meghnaghat Power Co. Ltd. & HSD & 305 \\
\hline 16. & Digital power \& Associates Gagnagar & F. Oil & 102 \\
\hline 17. & Baraka Patenga & F. Oil & 50 \\
\hline 18. & ECPV Chattogram Limited $108 \mathrm{MW}$ & F. Oil & 108 \\
\hline 19. & $\begin{array}{c}\text { Lakdhanvi lanka -Bangla Jangalia Cumilla } 52 \\
\text { MW }\end{array}$ & F. Oil & 52 \\
\hline 20. & $\begin{array}{l}\text { Sinha Peoples Energy Ltd. Katpatti 52.5 MW } \\
\text { Exp. }\end{array}$ & F. Oil & 51 \\
\hline 21. & Summit Barishal 110MW & F. Oil & 110 \\
\hline 22. & $\begin{array}{l}\text { Sumit Narayangonj Power Unit-2 Madangonj } \\
55 \mathrm{MW}\end{array}$ & F. Oil & 55 \\
\hline 23. & Dhaka (Doreen) Northan Power Ltd. Manikganj & F. Oil & 55 \\
\hline 24. & Dhaka (Doreen) Southern Power Ltd. Nobabgonj & F. Oil & 55 \\
\hline 25. & $\begin{array}{l}\text { Power Pac Mutiara Jamalpur } 95 \text { MW Power Plant } \\
\text { Ltd. }\end{array}$ & F. Oil & 95 \\
\hline 26. & $\begin{array}{c}\text { CLC Power CO. Ltd. 108 MW Bosila } \\
\text { Keranigonj } \\
\end{array}$ & F. Oil & 108 \\
\hline \multirow[t]{2}{*}{27.} & $\begin{array}{c}\text { Banco Energy Generation } 54 \text { MW, } \\
\text { Kamalaghat, }\end{array}$ & F. Oil & 54 \\
\hline & Munshiganj & & \\
\hline 28. & Aggreko, Brahmangaon 100MW & HSD & 100 \\
\hline 29. & APR Energy 300MW & HSD & 0 \\
\hline 30. & $\begin{array}{l}\text { United Mymenshingh Power Ltd.(UMPL) } \\
\text { 200MW, }\end{array}$ & F. Oil & 200 \\
\hline 31. & Aggreko, Aourahati 100MW & HSD & 100 \\
\hline
\end{tabular}




\begin{tabular}{|c|c|c|c|}
\hline SL No & Name of power plant & $\begin{array}{l}\text { Type } \\
\text { of fuel }\end{array}$ & $\begin{array}{c}\text { Installed Capacity (As of June) } \\
\text { (MW) }\end{array}$ \\
\hline 32. & Engreen Solar Power Plant3MW, Sharishabari & Solar & 3 \\
\hline 33. & Sailo Solar Power Plant Shantahar & Solar & 0 \\
\hline \multirow[t]{2}{*}{34.} & Shalla $400 \mathrm{KW}$ Solar & Solar & 0 \\
\hline & RENTAL \& SIPP & & \\
\hline 1. & Bogura RPP (24MW) 15 yrs GBB & Gas & 22 \\
\hline 2. & Bogura 20 RPP (3 Yrs) Energy Prima & Gas & 20 \\
\hline 3. & Ghorashal 78 MW QRPP (3 Yrs Max Power) & Gas & 78 \\
\hline 4. & Tangail SIPP (22 MW) (Doreen Power Ltd) & Gas & 22 \\
\hline 5. & Feni SIPP (22 MW) (Doreen Power Ltd.) & Gas & 22 \\
\hline 6. & $\begin{array}{l}\text { Jangalia } 33 \text { MW (Summit Purbanchol Po. Co. } \\
\text { Ltd. }\end{array}$ & Gas & 33 \\
\hline 7. & $\begin{array}{c}\text { Ashugonj } 55 \text { MW } 3 \text { Years Rental (Precision } \\
\text { Energy) }\end{array}$ & Gas & 55 \\
\hline 8. & B.Baria 70 MW QRPP (3 Yrs Aggreco) & Gas & 85 \\
\hline \multirow[t]{2}{*}{9.} & Ashugonj 53 MW Q. Rental PP (3 Years, United & Gas & 53 \\
\hline & Power) & & \\
\hline 10. & Kumargaon 50 MW 3 yrs (Energyprima) & Gas & 50 \\
\hline 11. & Shahzibazar 86 MW RPP (15 yrs) & Gas & 86 \\
\hline 12. & Shahzibazar 50 MW RPP (3 yrs) (Energyprima) & Gas & 50 \\
\hline 13. & Kumargaon 10 MW Desh Combridge (15 Yrs) & Gas & 10 \\
\hline 14. & $\begin{array}{c}\text { Fenchugonj 51 MW Rental (15 Yrs) } \\
\text { (Barakatullah) }\end{array}$ & Gas & 51 \\
\hline 15. & Fenchugonj 50 MW Rental (Energy Prima) & Gas & 44 \\
\hline 16. & Barabkundu SIPP 22 MW (Regent Power) & Gas & 22 \\
\hline 17. & Malancha, EPZ, Ctg & Gas & 0 \\
\hline 18. & Bhola 32 MW (Venture Energy Resources Ltd.) & Gas & 33 \\
\hline 19. & Ghorashal 45 MW (Aggreko) & Gas & 0 \\
\hline 20. & Ghorashal 100 MW RPP (Aggreko) & Gas & 0 \\
\hline \multirow[t]{2}{*}{21.} & Shahjahanullah Power Gen Co. Ltd. (REB, & Gas & 0 \\
\hline & Marchant) & & \\
\hline 22. & Aggreko 95 MW Bhola & Gas & 95 \\
\hline 23. & Khulna 55 MW RPP 3 yrs (Aggreko) & HSD & 55 \\
\hline 24. & Bheramara 110 MW 3 Yrs Rental (Quantum) & HSD & 0 \\
\hline 25. & $\begin{array}{c}\text { Khulna } 115 \text { MW QRPP (5 Yrs Summit) (KPCL } \\
\text { U-2) }\end{array}$ & F. Oil & 115 \\
\hline 26. & Noapara 40 MW QRPP (5 Yrs Khan Jahan Ali) & F. Oil & 40 \\
\hline 27. & Pagla 50 MW ( DPA & HSD & 50 \\
\hline \multirow[t]{2}{*}{28.} & Shiddirgonj 100 MW Q. Rental 3 Yrs (Desh & HSD & 100 \\
\hline & Energies) & & \\
\hline 29. & Madangonj 100 MW QRPP (5 Yrs Summit) & F. Oil & 102 \\
\hline 30. & Meghnagat 100 MW QRPP (5 Yrs) IEL & F. Oil & 100 \\
\hline 31. & $\begin{array}{c}\text { Siddhirganj } 100 \text { MW QRPP (5 Yrs) Dutch } \\
\text { Bangla }\end{array}$ & F.Oil & 100 \\
\hline 32. & Shikalbaha 55 MW Rental (3 Years) Energies & F. Oil & 51 \\
\hline 33. & Amnura 50 MW QRPP (5Yrs, Sinha Power) & F. Oil & 50 \\
\hline \multirow[t]{2}{*}{34.} & $\begin{array}{c}\text { Mutiara, Keranigonj } 100 \text { MW QRPP (5 Yrs) } \\
\text { Power }\end{array}$ & F. Oil & 100 \\
\hline & $\mathrm{Pac}$ & & \\
\hline 35. & Julda 100 MW QRPP (5 Yrs, Acron Infra) & F. Oil & 100 \\
\hline \multirow[t]{2}{*}{36.} & Katakhali 50 MW QRPP (ENA) & F. Oil & 50 \\
\hline & IMPORT & & \\
\hline 1. & Import from NVVN \& PTC (Bheramara) & Import & \\
\hline 2. & Import from Tripura 100MW & Import & \\
\hline \multirow[t]{2}{*}{3.} & Import from India $40 \mathrm{MW}$ & Import & \\
\hline & GRAND TOTAL & & 15,953 \\
\hline
\end{tabular}


Table-2: List of under-construction power plant $[2,3,5,11]$

\begin{tabular}{|c|c|c|c|}
\hline SL No & Name & Type & $\begin{array}{c}\text { Capacity } \\
\text { (MW) }\end{array}$ \\
\hline 1 & $\begin{array}{c}\text { Matarbari } \\
\text { Power } \\
\text { Station }\end{array}$ & Coal fired & 1200 \\
\hline 2 & $\begin{array}{c}\text { Payra } \\
\text { Thermal } \\
\text { Power } \\
\text { Plant }\end{array}$ & Coal fired & 1320 \\
\hline 3 & $\begin{array}{c}\text { Rampal } \\
\text { Power } \\
\text { Plant }\end{array}$ & Coal fired & 1320 \\
\hline 4 & $\begin{array}{l}\text { Feni } \\
\text { Lanka } \\
\text { Power } \\
\text { Limited }\end{array}$ & $\begin{array}{l}\text { Oil and } \\
\text { gas fired }\end{array}$ & 114 \\
\hline 5 & $\begin{array}{l}\text { Payra } \\
\text { LNG } \\
\text { Power } \\
\text { Plant }\end{array}$ & $\begin{array}{l}\text { Oil and } \\
\text { gas fired }\end{array}$ & 3600 \\
\hline 6 & $\begin{array}{c}\text { Rooppur } \\
\text { Nuclear } \\
\text { Power } \\
\text { Plant }\end{array}$ & Nuclear & 2,400 \\
\hline 7 & $\begin{array}{c}\text { Teknaf } \\
\text { Solar Park }\end{array}$ & Solar & 200 \\
\hline Total & \multicolumn{3}{|c|}{10154} \\
\hline
\end{tabular}

There is some proposed power plant. These power plant will start electricity generation within next few years. When all of proposed power plant start generation of electricity, we hope that there will no load-shedding occur.

Table-3: List of proposed power plant $[2,3,5,11]$

\begin{tabular}{|c|c|c|c|}
\hline $\begin{array}{c}\text { SL } \\
\text { No }\end{array}$ & Name & Type & $\begin{array}{c}\text { Capacity } \\
\text { (MW) }\end{array}$ \\
\hline 1 & $\begin{array}{c}\text { Sutiakhali, Mymensingh } \\
\text { Solar Park }\end{array}$ & Solar & 50 \\
\hline 2 & Sunamganj Solar Park & Solar & 32 \\
\hline \multicolumn{2}{|c|}{ Total } & 82 \\
\hline
\end{tabular}

In present, Bangladesh generate electricity about 15,042MW (including 8,845MW in public sector \& $6,197 \mathrm{MW}$ in private sector). Bangladesh government also import 660MW electricity from India. To fulfil our demand of electricity the 7554MW power plant are under construction and 2682MW power are proposed. At present, only $58 \%$ electricity generate by publicly and privately. About $29 \%$ power plants are under construction. After completing their construction about 7554 MW generation capacity add with our national grids.

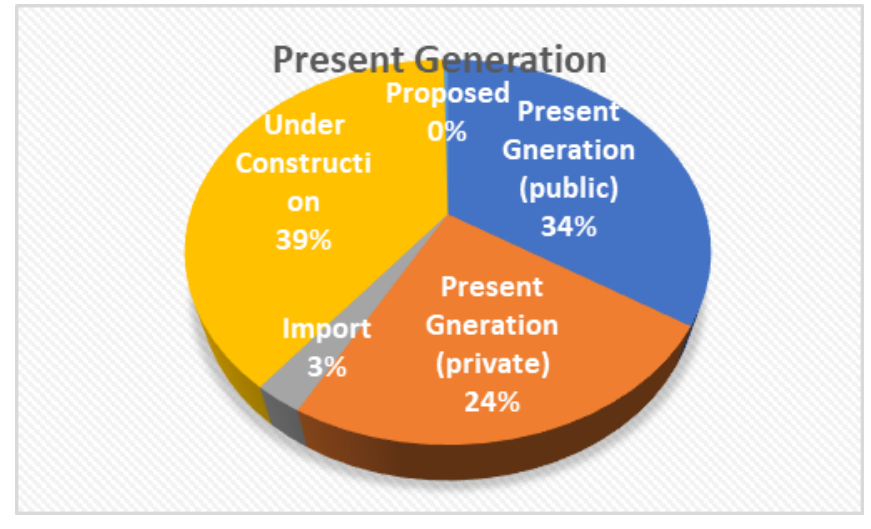

\section{B. Electrical Energy Demand and supply}

Demand and supply: Though electricity supply is less than the demand, this difference is closing gradually according to the government. After Bangladesh's independence in 1971 , only $3 \%$ of the total population had access to electricity. This ratio has gone up to $59.6 \%$ in 2012, and almost $76 \%$ by 2016.[2] The government have already taken some steps to increase electricity supply like Ruppur Nuclear Powerplant. $[2,3,5,7]$ The demand of electricity was $3900 \mathrm{MW}$ in 2000 and supply only $2030 \mathrm{MW}$. The demand is increase to $6454 \mathrm{MW}$ in 2010 and $14014 \mathrm{MW}$ in 2018. The supply also increases to $4200 \mathrm{MW}$ in 2010 and $8845 \mathrm{MW}$ in 2019. We determine some projection of demand and supply up to 2036. This projection show that we overcome from storage of electricity in 2034.

Table-4: Year wise demand \& supply of electricity in Bangladesh. [3,4,6,9]

\begin{tabular}{|c|c|c|}
\hline Year & $\begin{array}{c}\text { Grid system } \\
\text { Demand (MW) }\end{array}$ & Supply (MW) \\
\hline 2000 & 3900 & 2030 \\
\hline 2002 & 4000 & 2400 \\
\hline 2004 & 4300 & 3103 \\
\hline 2006 & 4730 & 3675 \\
\hline 2008 & 5569 & 4033 \\
\hline 2010 & 6454 & 4200 \\
\hline 2012 & 7518 & 4960 \\
\hline 2014 & 9268 & 6434 \\
\hline 2016 & 11405 & 7360 \\
\hline 2018 & 14014 & 8845 \\
\hline
\end{tabular}

Demand \& Supply Curve in Bangladesh (2000-2018):

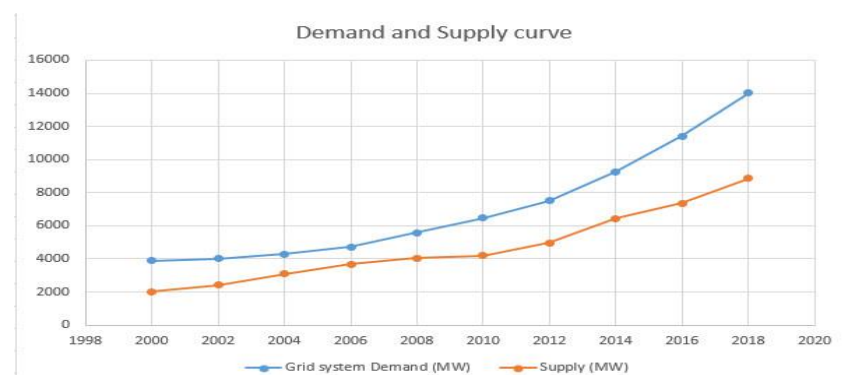


The demand and supply curve show that the shortage of electricity in present. So, some time we face load shedding. It also shows that we can fulfil our all demand in 2032. In 2035, we hope some extra electricity will generate after fulfil all demand.

\section{CONSUMPTION PATTERN OF BANGLADESH}

Most of the energy consumption occur in domestic and Industrial sector. [6] According to the Bangladesh Power Development Board Energy Consumption Pattern of our Country is given in table:

Table-5: All retail consumption:

\begin{tabular}{|r|r|r|l|l|l|}
\hline Year & Domestic & Agriculture & Commercial & Industrial & Others \\
\hline 2018 & $52.65 \%$ & $2.60 \%$ & $9.19 \%$ & $33.42 \%$ & $2.14 \%$ \\
\hline 2017 & $50.18 \%$ & $3.09 \%$ & $9.27 \%$ & $35.45 \%$ & $2.0 \%$ \\
\hline 2016 & $50.89 \%$ & $3.61 \%$ & $9.34 \%$ & $34.28 \%$ & $1.88 \%$ \\
\hline 2015 & $51.66 \%$ & $4.13 \%$ & $9.30 \%$ & $33.58 \%$ & $1.32 \%$ \\
\hline
\end{tabular}
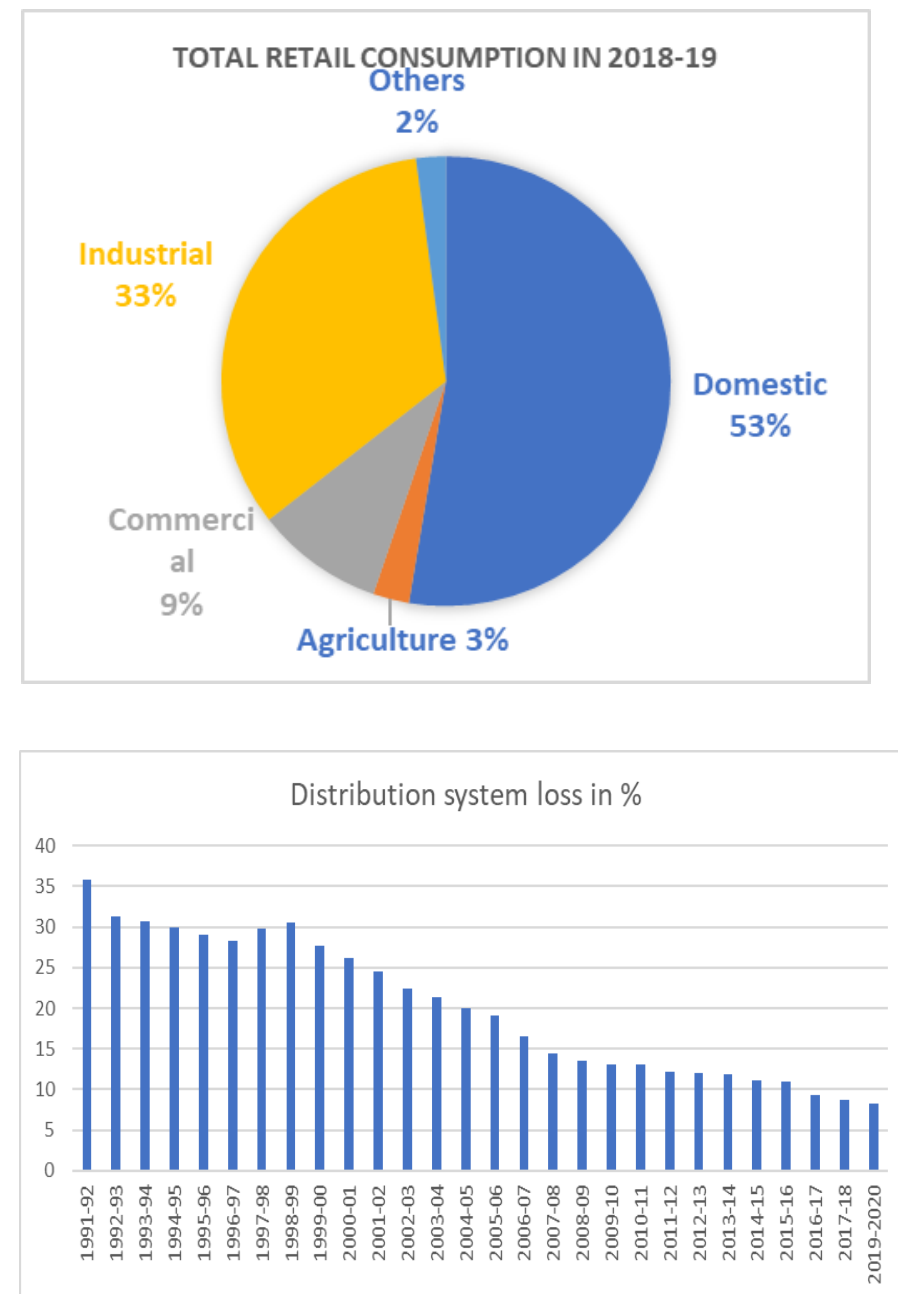

The table show that about $53 \%$ electricity consumption by domestic uses and 33\% electricity consumption by industrial uses and 0nly 14\% electricity used by Agricultural and commercial fields.

\section{DISTRIBUTION SYSTEM LOSS}

In 1991, Distribution loss in Bangladesh about $40 \%$ but present it reduce to only $10 \%$ in 2018 . $[2,3,6,13]$

Total system loss (Distribution and Transmission loss) last 12 years is given below:

Table-6: All system loss(2006-2018)[7]

\begin{tabular}{|c|c|c|c|}
\hline Year & $\begin{array}{c}\text { Distributi } \\
\text { on Loss } \\
(\%)\end{array}$ & $\begin{array}{c}\text { Transmissi } \\
\text { on loss } \\
(\%)\end{array}$ & $\begin{array}{c}\text { Total } \\
\text { System } \\
\text { Loss } \\
(\%)\end{array}$ \\
\hline 2006 & 19.06 & 4.97 & 21.25 \\
\hline 2007 & 16.58 & 3.99 & 20.25 \\
\hline 2008 & 14.43 & 2.9 & 18.45 \\
\hline 2009 & 13.57 & 2.52 & 16.85 \\
\hline 2010 & 13.10 & 2.24 & 15.73 \\
\hline 2011 & 13.06 & 2.18 & 14.73 \\
\hline 2012 & 12.15 & 2.35 & 14.61 \\
\hline 2013 & 11.95 & 2.75 & 14.36 \\
\hline 2014 & 11.89 & 2.72 & 14.13 \\
\hline 2015 & 11.17 & 2.74 & 13.55 \\
\hline 2016 & 11.01 & 2.73 & 13.10 \\
\hline 2017 & 10.92 & 2.71 & 12.74 \\
\hline 2018 & 9.89 & 2.76 & 11.87 \\
\hline
\end{tabular}

In 2006 , the total system loss $21.25 \%$ which is a large loss in our system. These losses can be reduced to $11.87 \%$ in 2018.

\section{TARGETS OF POWER GENERATION FROM RENEWABLE ENERGY}

The source of gas and fuel are limited that is why the government taken some step to use renewable source to generate electricity and already started some project such as Teknaf Solar Park, Sutiakhali, Mymensingh Solar Park, Sunamganj Solar Park.

Now Bangladesh generate only 550 MW electricity from renewable sources. Targets of power generation 
capacity by 2030 from renewable energy source given below: $[1,2,10,13]$

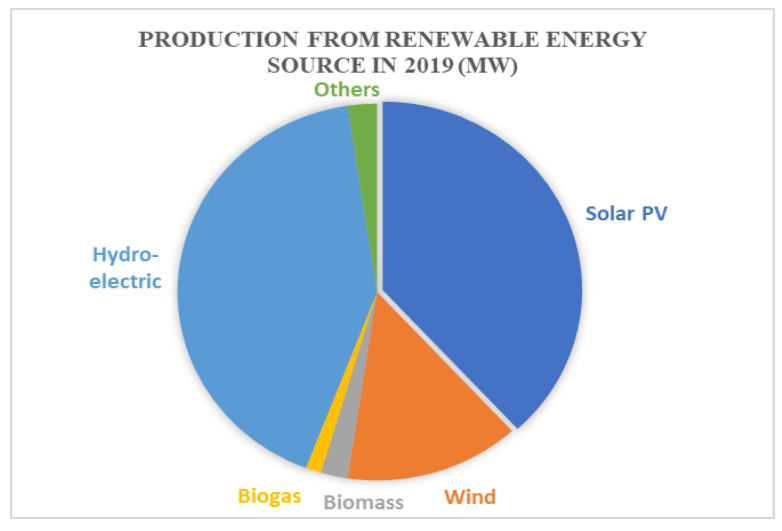

Table-7: Target use of renewable energy

\begin{tabular}{|c|c|c|c|}
\hline $\begin{array}{c}\text { Renewable } \\
\text { Energy } \\
\text { source }\end{array}$ & $\begin{array}{c}\text { Achievements } \\
\text { in 2019(MW) }\end{array}$ & $\begin{array}{c}\text { Target } \\
\text { production } \\
\text { in 2030 } \\
\text { (MW) }\end{array}$ & $\begin{array}{c}\text { Potential } \\
\text { (MW) }\end{array}$ \\
\hline Solar PV & 210 & 500 & 5045 \\
\hline Wind & 78 & 450 & 2000 \\
\hline Biomass & 12 & 50 & 274 \\
\hline Biogas & 7 & 10 & - \\
\hline $\begin{array}{c}\text { Hydro- } \\
\text { electric }\end{array}$ & 230 & 400 & - \\
\hline Others & 13 & 20 & - \\
\hline Total & 550 & 1430 & - \\
\hline
\end{tabular}

\section{Scope of Renewable Energy:}

Solar PV: Bangladesh is subtropical country. The daily solar radiation is $4-6.5 \mathrm{kWh} / \mathrm{m} 2$. So, it makes the use of solar panels very effective. The government introduced a scheme known as solar home systems (SHS) to provide electricity to households with no grid access.

Wind: The average wind speed in Bangladesh between $3-4.5 \mathrm{~m} / \mathrm{s}$. There is a good opportunity in island and coastal areas for the application of wind mills for pumping and electrification.

Biomass: The common biomass resources available in Bangladesh are agricultural crop residues, wood residues, animal waste and municipal solid waste, etc.

Biogas: Bangladesh has the potential to generate 5204 $\mathrm{Mm} 3$ of biogas which could add around $7.30 \mathrm{GWh}$ electricity to the national grid. The energy starved Bangladesh needs to promote biogas technology to reduce the dependence on conventional energy system as well to ensure energy security for the future.

Hydro-electric: Power can be produced through using the gravitational force of falling or flowing water. It is the most widely used form of renewable energy.The dam at Kaptaimukh, built on the River Karnafuli in the Chittagong Hill Tracts (CHT), is the only hydroelectric power project in Bangladesh.

\section{METHODOLOGY}

\section{NEWTON'S EXTRAPOLATION}

Extrapolation is a type of estimation, beyond the original observation range, the value of a variable on the basis of its relationship with another variable. The formula of newton's extrapolation s given by:

$$
\begin{aligned}
& Y_{n+1}=Y_{n}+\frac{Y_{n}-Y_{n-1}}{X_{n}-X_{n-1}} \times\left(X_{n+1}-X_{n}\right) \\
& >\mathrm{Y}=\text { Present demand/supply } \\
& >\mathrm{X}^{\mathrm{n}}=\text { Present Year } \\
& >\mathrm{Y}^{\mathrm{n}}=\text { Demand/Supply of Projection } \\
& >\mathrm{X}^{\mathrm{n}+1}=\text { Year of Projection }
\end{aligned}
$$

Demand and Supply projection

Newton's Extrapolation is linear extrapolation but our past data is not linear so we should be considering some factor such as transmission loss, Distribution loss, System losses, Target of renewable energy use and use of captive power. We consider decrement when calculated grid demand, Kd which depend on captive power use (such as personal solar, Biogas etc.) and consider increment when calculate grid supply, Ks which depend on reducing transmission loss, distribution loss and target of renewable energy used. For projection of demand,

$$
Y_{n+1}=Y_{n}+\frac{Y_{n}-Y_{n-1}}{X_{n}-X_{n-1}} \times\left(X_{n+1}-X_{n}\right)-K_{d}
$$

For projection of Supply,

$$
Y_{n+1}=Y_{n}+\frac{Y_{n}-Y_{n-1}}{X_{n}-X_{n-1}} \times\left(X_{n+1}-X_{n}\right)+K_{s}
$$

We determine some projection of demand and supply up to 2036. This projection show that we overcome from storage of electricity in 2034.

Table-5.1: projection of demand and supply up to 2036

\begin{tabular}{|c|c|c|}
\hline Year & $\begin{array}{c}\text { Grid system } \\
\text { Demand (MW) }\end{array}$ & $\begin{array}{c}\text { Grid Supply } \\
\text { (MW) }\end{array}$ \\
\hline 2020 & 16473 & 10590 \\
\hline 2022 & 18782 & 12505 \\
\hline 2024 & 20941 & 14620 \\
\hline 2026 & 22950 & 16935 \\
\hline 2028 & 24809 & 19450 \\
\hline 2030 & 26518 & 22165 \\
\hline 2032 & 28077 & 25080 \\
\hline 2034 & 29486 & 28195 \\
\hline 2036 & 30745 & 31510 \\
\hline
\end{tabular}




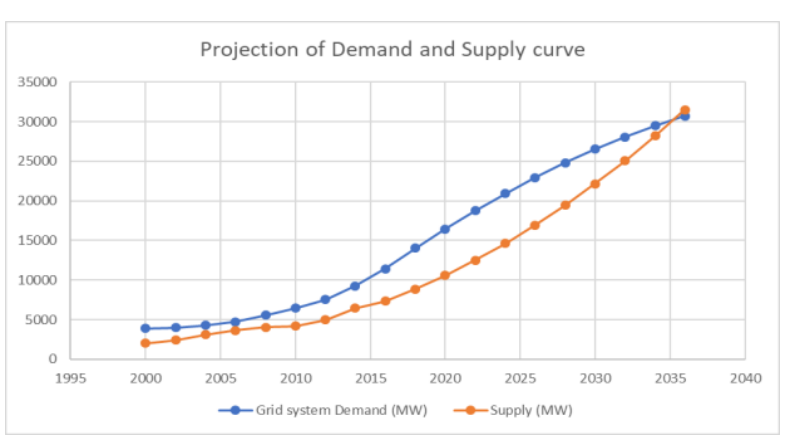

The demand and supply curve show that the shortage of electricity in present. So, some time we face load shedding. It also shows that we can fulfil our all demand in 2035. In 2036, we hope some extra electricity will generate after fulfil all demand.

\section{ACKNOWLEDGMENT}

First of all, we would like to express our heartiest thank to almighty Allah to give us blessing and opportunity to complete this project successfully. Then we express our gratitude and thank to our honorable project supervisor Professor Dr. B. K. Bala, Dean, Faculty of Engineering, BSMRSTU for sharing his absolute expertise and valuable time. We are also grateful to different online sources from which we collect a lot of information.

\section{CONLCUTION}

In this paper, we have briefly discussed about electrical energy situation in Bangladesh-demand, supply and future projection. According to the research, total electricity supply is almost $15000 \mathrm{MW}$ whether total demand is more than supply [3]. The government have taken some steps to balance this demand and supply. We also projected energy generation till 2036 regarding to the generation improvement. We have calculated that the total grid power generated in Bangladesh is $8845 \mathrm{MW}$ and total captive power generated is 6197 MW [1,3]. As the energy sources are limited, renewable energy sources are also been used.

If we use of captive power, reduce system, distribution and transmission loss and use targets of Renewable Energy, we hope to solve shortage of electricity and our projection will be successful.

\section{REFERENCES}

[1] B.K. Bala M.S. Alam, Md. Alam Hossain Mondal, M.K. Rowson,Energy Perspective of Greenhouse Gas Emission for Transition from Non-renewable to Renewable Energy: The Case of Bangladesh.
[2] Bangladesh Energy Situation, energypedia.info

[3] Annual report of Bangladesh Power Development Board 2017-18, Published in June 2019.

[4] Moazzem,Ali, "The Power and Energy Sector of Bangladesh: Challenges of Moving beyond the Transition Stage" 10 March, 2019

[5] BDNews24,(2016, August 20), Bangladesh protesters announce fresh demonstration against Rampal Power Plant.

[6] Bangladesh Power Development Board, "An Overview of Power Sector of Bangladesh" November, 2011

[7] Power Division,Bangladesh, "Power System Master Plan 2019."

[8] Electricity sector in Bangladesh - Wikipedia,

[9] "Present Installed Generation Capacity (MW) as on 30 June, 2018". Bangladesh Power Development Board. Retrieved 17 July 2018.

[10] Rahman and Kholilullah (October 2017). "Use of Solar Panel at Rural Areas in Bangladesh: Impacts, Financial Viability and Future Prospects" (PDF). International Journal of Science and Research (IJSR). Volume 6 Issue 10.

[11] List of power stations in Bangladesh - Wikipedia.

[12] Saiful Islam, Md. Ziaur Rahman Khan, "A review of energy sector of Bangladesh" 1st International Conference on Energy and Power, ICEP2016, 1416 December 2016, RMIT University, Melbourne, Australia.

[13] Golam Kibria, "Power Sector OverviewBangladesh"'Integrated Research and action for Development. 